\title{
Flatworms (Platyhelminthes) Associated with Slaughtered Cows in Abattoirs Found in Bali Town Taraba State Nigeria
}

\author{
C. A. Ukwubile ${ }^{1 *}$, M.S. Bingari ${ }^{2}$ \\ ${ }^{1}$ Medicinal Plants Research Laboratory, Central Academic Center/Sancta Maria Clinic Inte grated Laboratory \\ Bali, Nigeria \\ ${ }^{2}$ Department of Biological Sciences, Taraba State University Jalingo, Nigeria
}

*Corresponding Author: C. A. Ukwubile, Medicinal Plants Research Laboratory, Central Academic Center/Sancta Maria Clinic Integrated Laboratory Bali, Nigeria

\begin{abstract}
:
Background: Flatworm infections are among the most common type of infections occurring mostly in underdeveloped and developing countries resulting in malnutrition and deaths is some cases.

Aim: This study was carried in order to determine the type offlatworms often associated with cows slaughtered in Bali abattoirs with a view to knowing their prevalence rate.

Method: Collection of samples took place in the morning hours from 6:30 am-7 am daily during the sampling periods from January 2017 to December 2017. A total number of 140 samples were analyzed by ultracentrifugation, staining and microscopy techniques.

Results: The infection rate was found to be higher in male cows than in female cows. The results further showed that flatworm infestation in the infected cows was as a result of poor water, improper livestock management, and contamination of the cow's food with cyst of helminthes. Fasciola hepatica infestation was highly prevalence when compared with Taenia saginata with the lowest percentage prevalence rate.

Conclusion: The study therefore, showed that Fasciola hepatica and Taenia saginata were the only dominant in male cows with $72 \%$ prevalence rate while the female cows have the least prevalence rate of $F$. hepatica and T. saginata infections. In all, Fasciola hepatica had the highest incidence rate leading to fasciolliasis in the cows and in some cases human.
\end{abstract}

Keywords: Flatworms, Platyhelminthes, Taenia saginata, Fasciola hepatic, Slaughtered cows, Bali.

\section{INTRODUCTION}

Parasitic Platyhelminthes infection is still posed serious public health problems in the world. It is estimated that about 1.5 billion people are affected, and 400 million are ill as a result of these infections [1]. The high prevalence rate in young people is attributed to many factors, particularly the social and economical situation of the individuals, which is the main cause of this high prevalence of parasitic Platyhelminthes, as well as unkempt environment, poor sanitation and lack of personal hygiene [2].

Parasitic Platyhelminthes infections are among the most common infections occurring throughout the developing world. The main transmission routes for most of the parasitic Platyhelminthes are fecal-oral, contaminated foods or water. Parasites such Entamoeba histolytica, Ascaris lumbricoides (hookworms), Necator americanus, Ancylostoma duodenale, and whipworm (Trichuris trichiura) are the major causes of water and food-borne disease globally [3]. Such infections are linked to lack of sanitation, lack of access to safe water and improper hygiene as well as poverty. These infections deprive the poorest of the good health thereby contributing to economic instability and social marginalization. The poor people of undeveloped nations experienced a cycle where under nutrition and repeated infections lead to excess morbidity that can continue from generation to generations. People of old ages are infected by this cycle of prevalence parasitic infections, especially communalized area such as villages and farm settlements [4].

A number of Platyhelminthes are natural parasites of man. The parasite form termed helminthes infections may evoke especially low level of infection. In heavy infections, symptoms as entirely non- 
pathogenic. A heavy helminthes load results from repeated infections because, as a rule, helminthes do not multiply inside their definite hosts and infect new animals before they can develop to maturity. There are few exceptions to this general rule, for example, the egg of Hymenolepis nana (the dwarf tapeworm of man), can also hatch and developed to adult in the human gut.

The world health organization (WHO) estimated that more than one billion of the world's population is chronically infected with soil-transmitted helminthes (STH). The high prevalence rate of these infections is closely corrected with poverty, poor environmental hygiene and impoverished health services [5]. These infections occur in all region of Africa and its public health importance has been demonstrated by a number of studies both in Nigeria [6-9], and elsewhere [10].It is endemic in both rural and urban population and it is posing a great threat to public health in developing countries .It has been shown to cause lack of appetites, intestinal abnormalities, poor absorption or increased loss of nutrient, which may result in protein-energy malnutrition and anemia. Global estimated prevalence rate of anemia in hookworm infection and a cognitive as well as improvement of infection, indicate that for each parasite, 10-50 million persons may be infected [11].

These parasite are widespread in the environment and major disease out-breaks have occurred as a result of contaminated drinking water and food. Recently, it has been estimated that one quarter of the world's populations is infected with one or more parasites .Ascaris lumbricoides, Trichuris trichiura and hookworms collectively referred to as soil-transmitted helminthes are the most common intestinal parasites .Ascaris lumbricoides is the largest and most common nematode parasitizing the animal as well as in human intestine and currently infects about one billion people worldwide [12].

This study was carried out in order to determine the type of flatworms found in slaughtered cows at Bali abattoir.

\section{Materials AND Methods}

Most of the materials and apparatus used throughout the study were provided for by the laboratory where this study was largely carried out, while others were purchased from the suppliers. The materials and apparatus used in the study include: hand gloves, forceps, razor blade, hand lens, sterilized universal container, microscope slides, scanning biological microscope, cotton-wool, normal saline ( $3 \mathrm{~mL}$ by 10 $\%$ volume),pasture pipette, applicator stick, etc. All glassware, slides, forceps, and universal conta iners were sterilized using an autoclave at $121^{\circ} \mathrm{C}$ for $30 \mathrm{~min}$.

\subsection{Specimen Collection}

The samples were collected from the liver and intestine of slaughtered male and female cows that were slaughtered by 7 am daily. Samples were collected in small sterilized polythene bag and then taken to the laboratory immediately and stored in the refrigerator at $-4{ }^{\circ} \mathrm{C}$ for further use. Sample collection was done each morning for one week in randomly selected months. A total number of five (5) cows consisting of 3 males and 2 females were slaughtered on daily basis according to the demand of beef by Bali people. A total of 140 samples were collected during the sampling periods.

\subsection{Samples Analysis}

A drop of normal saline ( $3 \mathrm{~mL}$ of $10 \%$ volume) was placed on grease free side using pasture pipette. About $0.5 \mathrm{~g}$ portion of the liver, intestine was placed on the slide using applicator stick after ultracentrifugation at $1000 \mathrm{rpm}$. This was mixed properly and then observed using a scanning biological microscope under $400 \mathrm{X}$ objectives, and observations of cyst and eggs were recorded.

\subsection{Data Analysis}

Data generated was subjected to simple percentage to determine the differences in terms of abundance of the species of the Platyhelminthes.

\section{RES ULTS AND Dis CUSSION}

The results for the prevalence rate of Platyhelminthes associated with slaughtered cows in our study was as shown in tables 1 and figures $\mathrm{i}$-ii below. The beef used in this study is an important commercial commodity due to its protein values, and also due to its inexpensive nature. As a results of this, beef are widely consumed in Bali town and its neighboring communities and villages. The knowledge of the type of Platyhelminthes in slaughtered cows is necessary for effective safe guard against consumption 
of the improperly cooked meat usually contaminated with the eggs and cysts of Platyhelminthes. Platyhelminthes burdens were high in the months of December (36\%) and April (27\%) (Table 1). This is could be due poor grazing by the cows during these months as a result of lack of pasture or foliage for grazers, thus, making the animals prone to helminthes infection.

Table1. Flatworm prevalence in slaughtered cows in Bali abattoir from January to December 2017

\begin{tabular}{|l|c|c|c|c|c|c|}
\hline \multicolumn{7}{|c|}{ Slaughtered cows with flatworms (male/female) } \\
\hline Flatworm & JAN & APR & AUG & DEC & \% Prevalence & Total (\%) \\
\hline Fasciola hepatica & $8 / 3$ & $9 / 6$ & $7 / 9$ & $9 / 9$ & $33 / 27$ & 50.81 \\
\hline Taenia saginata & $6 / 6$ & $8 / 4$ & $11 / 4$ & $7 / 11$ & $32 / 25$ & 48.31 \\
\hline \multicolumn{7}{|c|}{ Total worms = 118 } \\
\hline
\end{tabular}

Results are sampling of the total number of cows slaughtered in each of the sampled months.

The result of our study showed that parasitic Platyhelminthes were highly present in the samples analyzed within this periods (Table 1). It further showed that helminthes such as Fasciola hepatica and Taenia saginata were present in the as seen from the presence of their eggs and cysts (Plate i a-c), with $F$. hepatica being the most prevalence. This could be attributed to the ubiquitous nature of the parasites. Besides, meat is rich in nutrient and this may be susceptible to the helminthes for their own metabolic activities.

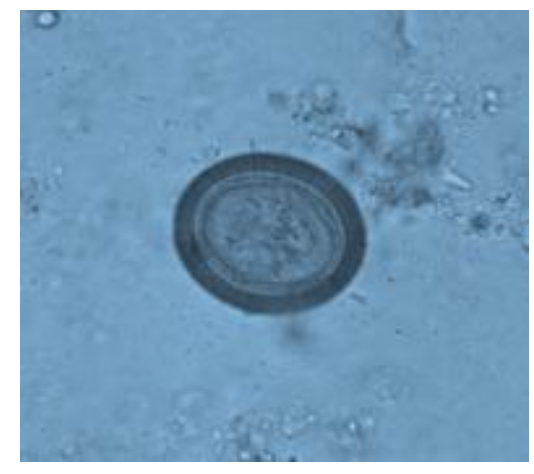

a. Unstained egg of Taenia saginata

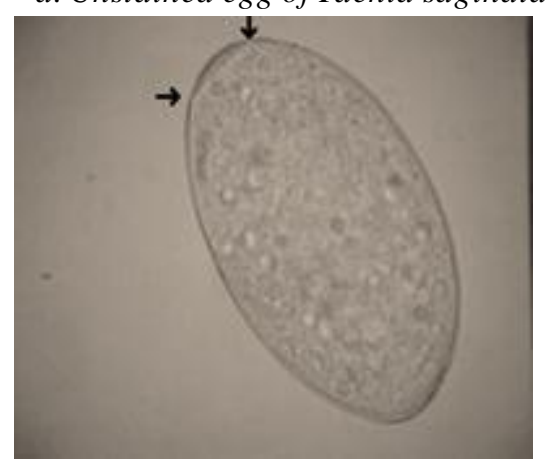

c. Stained egg of Fasciola hepatica in slaughtered $\delta$ and $q$ cows respectively

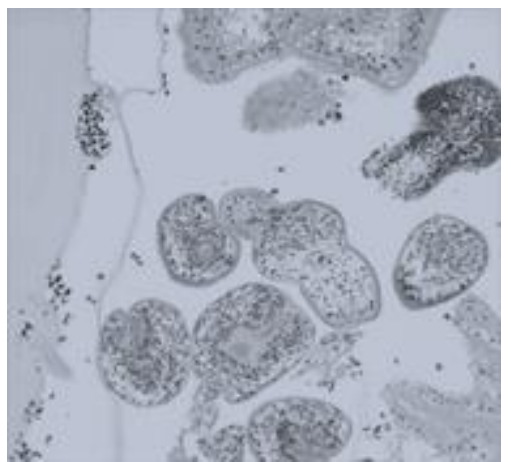

b. Hydatid cyst of T. saginata $400 X$

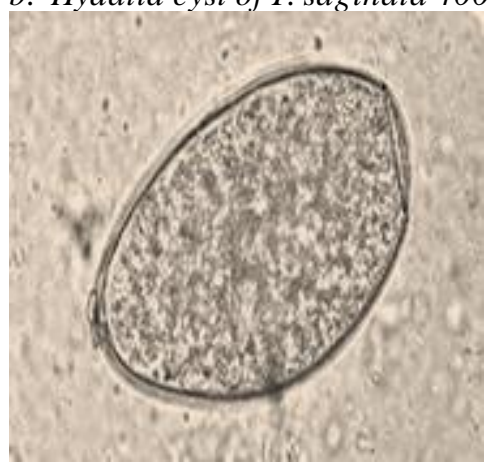

Plate i: Microscopic features observed in collected samples from Bali abattoir $400 X$

The abundance of these helminthes could be due to the fact that they produced spores which help to distribute them widely in their environment, thereby contaminating foods such as grasses fed upon by these cows. Beside, their eggs are covered with cuticle which help them resist harsh climatic condition of their environment. The presence of parasitic Platyhelminthes in the slaughtered beef may be as a result of improper maintenance and care of slaughtered cows. This was due to the fact that most of the butchers were not knowledgeable especially in animal cares. The study also showed that $T$. saginata infestation was prevalence in weeks 2 and 3. Taenia saginata for instance, obtained its food by absorption across the tapeworm's flat body membrane. Carbohydrates from the host are the most important for T. saginata to absorb in the form of polysaccharides. This form is easiest for the worm to breakdown into the usable form of glucose. It is possible that the parasite adopt this mechanism in the cows, and when the animals were slaughtered, the parasite continues its life cycle in humans when improperly cooked beef is eaten by the human's example roasted "suya" meat in most northern states of Nigeria. 
Fasciola hepatica was the most prevalence flatworms in the slaughtered cows (Table1, Figure i and ii). The adult $F$. hepatica develop from metacercaria (Plate ic) after they penetrate through gut and lodge in the liver and then gall-bladder and bile duct. The gall-bladder and duct may become distended, fibrotic and calcified. Large numbers migrating through liver cause hemorrhage causing a disease known as fascioliasis in the cows and sometimes in humans who subsequently eat not properly cooked beef. This adversely affects the quality of meat production. It has a sucking pharynx and a blind gut (no anus). It sucks in bile and inflammatory exudates and digests this. This may be due to the location of parasite in the liver as well as adaptation in its environment [15]. Both helminthes were seen to be prevalence in male cows than the female cows.

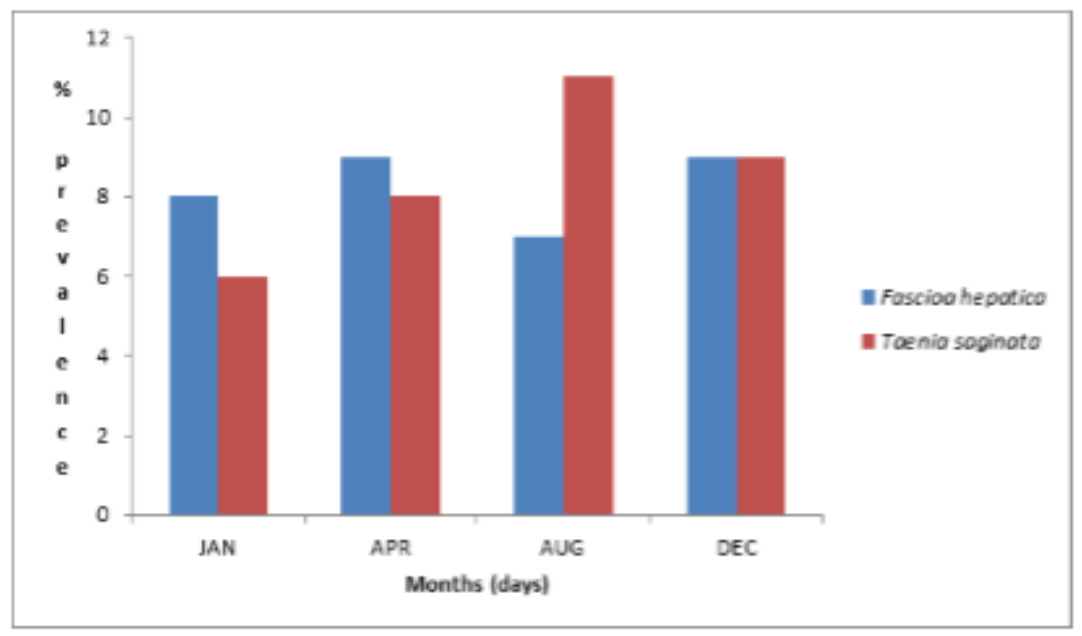

Figure1. Flatworm prevalence according to the months of slaughtering

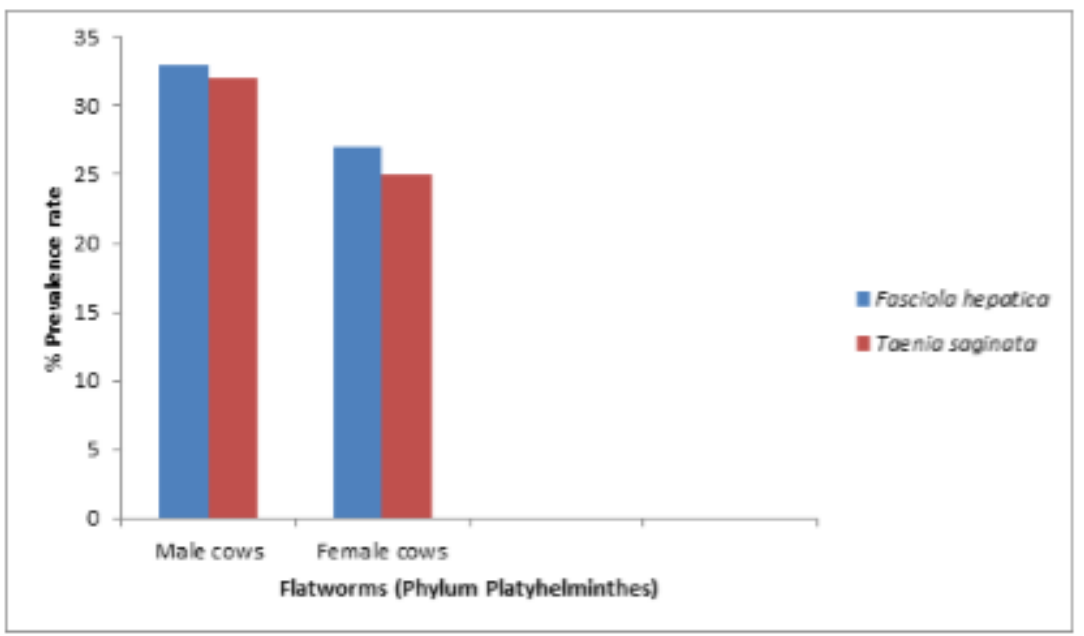

Figure2. Flatworm prevalence according to the sexes of the cow

This may be due to the fact that the body physiology of the male cows favors advance parasitaemia than the female cows or because of excessive deposition of fat in the adipose tissue of male cows than the females. The result may not be different from the above observation which correlates with the findings of various researchers [13-18]. This report might be due to improper cleaning of cow's surroundings and contaminated food as well as water.

\section{CONCLUSion}

Helminthes parasite infection in slaughtered cows in Bali abattoir is quite high with Fasciola hepatica (liver fluke) being the most prevalence. This was due to improper disposal of human wastes which resulted in the pollution of the surrounding environment where the animals grazed, and lack of adequate animal care of the environment where the cows usually grazed before they are taken to local markets for sale. This is also complicated by various unhygienic practices by people living in Bali town. Base on the findings from this study, the following recommendations were put forward: meat should be 
properly cooked before eating so as to destroy the cyst of these helminthes, slaughtering and rearing of cows should be done in hygienic environments by skilled persons to prevent flatworm infection.

\section{ACKNOWLEDGEMENT}

The authors are grateful to Bali slaughters Association for allowing us access to the samples at the abattoir.

\section{REFERENCES}

[1] Adeyeba,O and Odebumi, J.F (2007). Intestinal Helminthiasis among people in Vom, Plateau State, Nigeria. Parasitology; 1:39-42.

[2] Ahmed, B.G, Bhatti, M.A, and Malla, N (2002). Human toxocariasis and Ascariasis concomitant parasitism in strinagar, Kashmir, India. Indian Journal of Pathology and microbiology; 45:315-318.

[3] Aken 'Ova. E (2013). Gastro intestinal parasites of West African lung fish. Annals of vertebrate. Zoology, 5 (2):88-98.

[4] Al-Barway, S.E (1978).A survey on liver infection with F. gigantica among slaughtered animals in Iraq. Bull end Dis; 18:75-95.

[5] Chakravarty, $\mathrm{N}$ (2001). To bring about proper coordination in the street food sector and assume advocacy programme.A strategy Document.Pre totia Tcp/SAF/8924.

[6] WHO website: http://www.who.int/neglected_diseases/diseases/fascioliasis/en/index.html

[7] Dewest H.F and Townsen K.G (1990). Nzvet: 63-145.

[8] Eisnitsical A (1997).Slaughtered house the shoal. King strong if Gread. Neglect are in Human treatment inside the US meat industry peonetheus Book

[9] Fallah, M, Azimian, M.T and Hojati, M (2004). Epi Morphological study of Ascaris miusis M Hamadomcity, West of Iran Science Journal of Harmatan University medical Science; 11:55-60.

[10] FAO (2003). Assuring food safety and quality: Guideliness for strengthening National Food control system. Food and Nutrition Paper Number No 76.

[11] Luka, S.A, Ajaji I, and Umih, J.U (2000). Helminthiasis Among primary school age children in State, Nigeria. Journal of Parasitology; 21:109-116.

[12] Nock,I.H (2012). Parasitic plasmodium of unidentified germs in Kargo.Journal of Parasitology; 3(4):6168.

[13] Norhayati M, O Auuman P, Fatmah M.S. (1998). Some risk. Factors of Ascaris (Oranhashi) Children, medical journal of Malaysia. 53:401-410.

[14] Oyerinde, I.P.O (1999). Essentials of Tropical medical Parasitology (Ed) University press Ibadan: 213-238.

[15] R and Torimiro, S.E.A (1989). The epidemiology of Ascaris lumbricoides and other soil transmitted Helminthes in primary school children from Ile-ife Nigeria. Parasitology; 99:275-285.

[16] Salaka, A.A (2001). Effect of portable, water availability on intestinal parasitism .Among rural people with sewage disposal facilities in this injidurn and owutu sub-urban community of Lagos State Nigeria medical Practitioner; 42:276-341.

[17] Stephenson, L.S lathan, M.C and Ottersen,E.A (2000). Malnutrition and parasitic Helminthes infection on. Parasitology; 121:23-28.

[18] WHO (2003). Controlling disease due to helminthes infection in the world. In: World Health Organization Bulletin, Geneva: 61-62.

Citation: C.A. Ukwubile \& M.S. Bingari, "Flatworms (Platyhelminthes) Associated with Slaughtered Cowsin Abattoirs Found in Bali Town Taraba State Nigeria", International Journal of Research Studies in Biosciences (IJRSB), vol. 6, no. 3, pp. 18-22, 2018. http://dx.doi.org/10.20431/2349-0365.0603004

Copyright: ( $) 2018$ Authors. This is an open-access article distributed under the terms of the Creative Commons Attribution License, which permits unrestricted use, distribution, and reproduction in any medium, provided the original author and source are credited. 